Editorial

\title{
The Zika Virus Epidemic Four Years After: Where are We?
}

\author{
Marco Aurélio Palazzi Sáfadi, MD, $\mathrm{PhD}^{1}$ Flavia J. Almeida, MD, PhD ${ }^{1}$ \\ ${ }^{1}$ Department of Pediatrics, Santa Casa de Sao Paulo School of Medical \\ Sciences, Brazil
}

Am J Perinatol 2019;36(suppl S2):S4-S8.

Zika virus (ZIKV), an emerging arthropod-borne, singlestranded RNA virus, member of the Spondweni serocomplex (genus Flavivirus, family Flaviviridae), was first isolated in 1947 from a sentinel (monkeys held captive with the purpose of identifying yellow fever activity) rhesus monkey in a forest in Uganda. After its discovery, ZIKV was associated only with few sporadic mild cases in humans in Africa and Asia over the next 60 years. ${ }^{1}$ However, since 2007, when the first outbreak of ZIKV outside Africa and Asia was reported in the Federated States of Micronesia (Yap), it has been identified in subsequent outbreaks in French Polynesia, Pacific islands, and more recently, from 2015, Brazil was the first country in the Western Hemisphere to confirm autochthonous transmission of ZIKV associated with an outbreak of "dengue-like syndrome" cases. ${ }^{2}$

Interestingly, the potential for severe neurological outcomes after ZIKV infection was reported only after the outbreaks in French Polynesia, in 2013, and subsequently in Brazil. Furthermore, the most striking finding, the established relationship between ZIKV infection during pregnancy and a devastating congenital syndrome, was identified for the first time during the ZIKV outbreak in Brazil, leading to a dramatic increase in newborns with severe congenital malformations associated with ZIKV. ${ }^{2}$ The unique characteristics of the ZIKV outbreak in Brazil, where the population was completely susceptible (naïve) to the virus, affecting highly populated urban poor areas with high density of Aedes aegypti, and the established surveillance reporting system, are possible reasons to explain why the role of the ZIKV as a potential cause of congenital disease has only been recognized after circulation in Brazil. After the reports from Brazil raised a causal relationship between ZIKV infection in pregnancy and microcephaly and other congenital malformations; a retrospective analysis conducted in French Polynesia found an association between ZIKV and microcephaly. ${ }^{3}$

Although it is still unknown whether ZIKV infection provides life-long immunity, it is expected that in endemic places in Africa and Asia, where the virus is circulating for decades, a proportion of the women in childbearing age is likely to have been infected during childhood, limiting the number of susceptible women. It is also possible that the more severe out-

comes of ZIKV infection, observed in Brazil and other countries, may be related to genome mutation in virulence characteristics of the ZIKV circulating strain or even immune interaction between consecutive Flavivirus infections.

The ZIKV outbreak spread very rapidly in the Americas after the outbreak in Brazil. ${ }^{2}$ As of March 2019, 89 countries and territories reported current or past local transmission, among which several have reported microcephaly and/or central nervous system (CNS) malformation cases and Guillain-Barré Syndrome (GBS), potentially associated with ZIKV infection. Uruguay is currently the only country in the Americas, with evidence of established competent vector, but no known documented transmission of ZIKV ${ }^{4}$ ( - Fig. 1).

Brazil was the most affected country in the Americas, reporting 216,207 probable cases in 2016; 17,594 cases in $2017 ; 8,104$ cases in 2018 ; and by week 10 , in 2019 , only 2,062 cases. $^{5}$ The substantial decline in cases of ZIKV infection reported in the last 2 years is probably a result of a combination of "herd immunity" of the population, that became immune after being infected in previous years, reducing the number of susceptible, naive subjects and, thus, limiting the transmission of the virus in the community, together with a cyclical natural pattern, common to other Flavivirus like dengue that ebbs and flows in periodic waves.

Since 2015, the Ministry of Health in Brazil confirmed 3,332 cases of microcephaly and/or CNS malformation associated with ZIKV infection, with the majority occurring between 2015 and 2016 in the Northeast region. ${ }^{6}$ In those places, a common feature of the epidemic was the fact that most of the cases and the associated complications occurred in the poor urban areas, characterized by limited sanitary infrastructure, crowding, and poor quality of housing conditions that clearly facilitate the transmission of the virus by the A. aegypti mosquitoes. ${ }^{7}$

\section{Transmission}

ZIKV is primarily transmitted to humans by A. aegypti mosquitoes (less frequently by other Aedes species), the same vector

Copyright $\odot 2019$ by Thieme Medical Publishers, Inc., 333 Seventh Avenue, New York, NY 10001, USA. Tel: +1(212) 584-4662
DOI https://doi.org/ 10.1055/s-0039-1691769. ISSN 0735-1631.

Marco Aurélio Palazzi Sáfadi, MD,

PhD, Department of Pediatrics,

Santa Casa de Sao Paulo School of

Medical Sciences, Alameda dos

Indigenas, 228, São Paulo

04059 060, Brazil

(e-mail: masafadi@uol.com.br). 


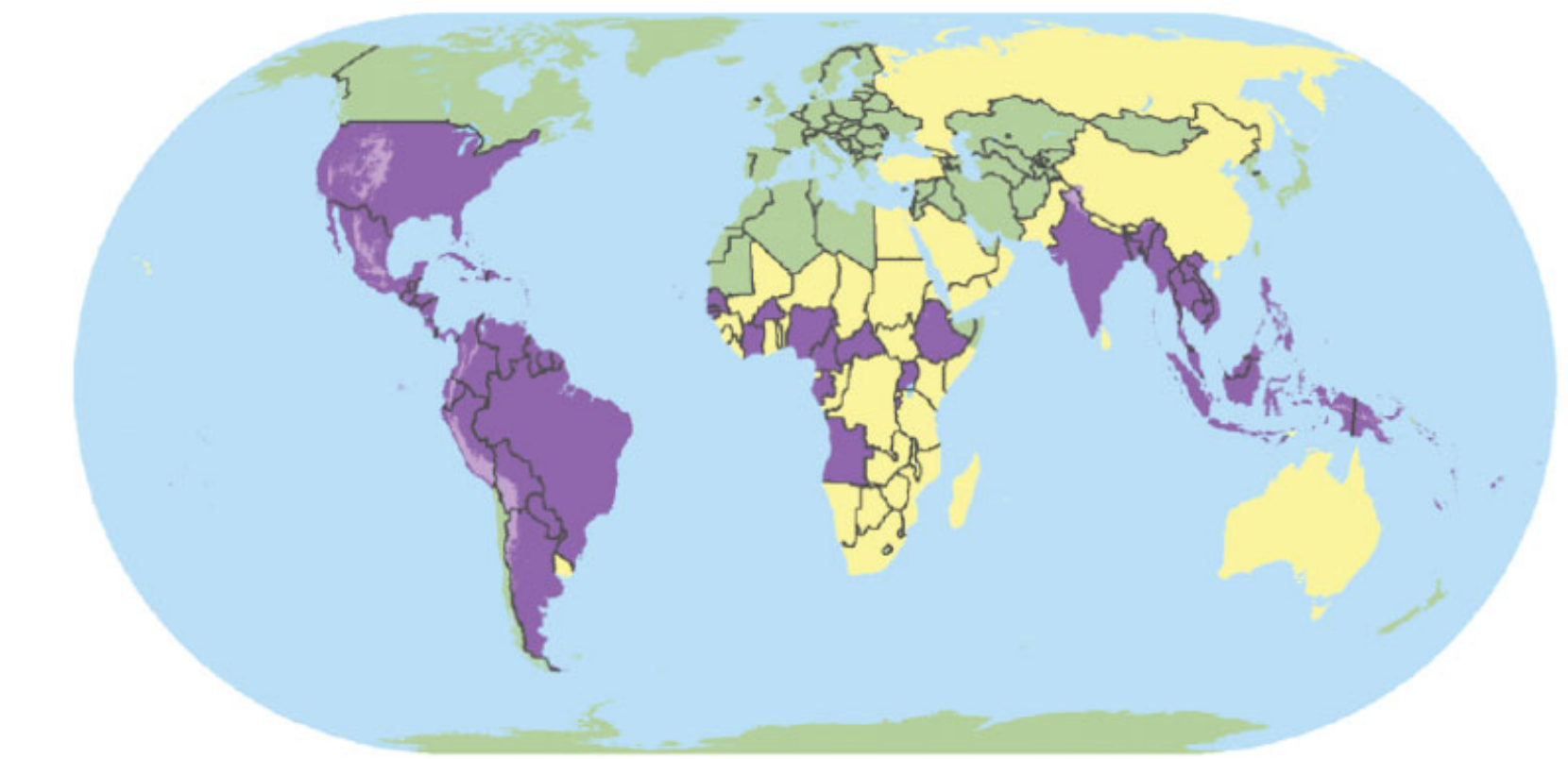

Country or territory that has ever reported Zika case $^{\star *}$ (past or current)

Areas with low likelihood of Zika infection because of high elevation (above 6,500 feet/2,000 meters)

Country with mosquito* but no reported Zika cases** Country or territory with no mosquitoes that spread Zika

* Aedes aegypti

** Locally acquired, mosquito-borne Zika cases

Fig. 1 World map of areas with risk of Zika. ${ }^{4}$

that can transmit yellow fever, dengue, and chikungunya. Infected people, both symptomatic and asymptomatic, can transmit ZIKV to mosquitoes throughout the viremic period that usually ranges from a few days to 1 week. Human and nonhuman primates are the main reservoirs of the virus, with humans acting as the primary host. ${ }^{1,2,4}$

Other nonvector-borne modes of transmission have been identified, including perinatal, in utero, sexual, blood transfusion, and laboratory exposure. ${ }^{1,2,4}$

Intrauterine transmission of ZIKV was confirmed in Brazil by the detection of virus genome in amniotic fluid samples of women with symptoms of ZIKV infection during the first trimester of pregnancy, whose fetuses have been diagnosed with microcephaly, in placental tissues from early miscarriages, and also in the blood and brain tissue of infants with congenital neurologic anomalies, including microcephaly. ${ }^{1,2,4}$

Based on reports that found detectable ZIKV RNA in semen for months after viral clearance from blood the Centers for Disease Control and Prevention (CDC) has issued guidelines, including the recommendation that any man who might have been exposed to Zika avoid conceiving for at least 3 months after his symptoms start. ${ }^{4}$

\section{Diagnosis}

Clinical diagnosis is limited by the nonspecific signs and symptoms of ZIKV infection, which are similar to other arboviral infections (e.g., chikungunya and dengue), are common in endemic areas. Abnormal laboratory findings, including mild thrombocytopenia, leukopenia, and elevations in acutephase markers of inflammation, serum lactate dehydrogenase, or liver transaminases have been observed in symptomatic patients. ${ }^{2,4}$

ZIKV specific diagnosis in nonpregnant symptomatic individuals is primarily based on the detection of ZIKV RNA by reverse transcription polymerase chain reaction (RT-PCR) performed on serum and/or urine specimens collected $<14$ days after onset of symptoms. ZIKV-specific immunoglobulin-M (IgM) and neutralizing antibodies can be detected by enzyme-linked immunosorbent assay in serum specimens collected by the end of the 1 st week of illness and up to 12 weeks postonset of symptoms. As the immune response develops, IgM titers rise in peripheral blood and the level of viral RNA generally declines. Serum IgM antibody testing should be performed if the RT-PCR result is negative or when $\geq 14$ days have passed since illness onset. IgG antibodies develop within days after IgM and can be detected for months to years. However, false-positive results due to crossreaction with related flaviviruses (e.g., dengue and yellow fever viruses) are commonly observed. ${ }^{2,4}$

Positive results in primary Flavivirus infections should be confirmed with a four-fold increase in the titer of neutralizing antibodies to ZIKV with plaque reduction neutralization test (PRNT). In endemic areas, where a great proportion of the population may have been previously infected with other flaviviruses or vaccinated against a related Flavivirus 
(i.e., secondary Flavivirus infection), neutralizing antibodies might still yield crossreactive results in these individuals. ${ }^{2,4}$

For infants with possible congenital ZIKV infection recommended testing includes evaluation for ZIKV RNA in infant serum and urine and ZIKV IgM antibodies in serum. ${ }^{4}$ Although the presence of ZIKV RNA is expected to be present only within the first few days after birth, persisted shedding in a congenitally infected infant for more than 2 months was reported..$^{8}$

\section{Clinical Manifestations}

Symptomatic infection, estimated to occur in less than $25 \%$ of the infected persons, is associated with a mild, self-limited disease, lasting few days, and characterized by low fever, pruritic rash, edema of extremities, conjunctivitis, headache, arthralgia, myalgia, and less commonly gastrointestinal symptoms, retro-orbital pain, and lymphadenopathy. ${ }^{2,4}$

\section{Neurological Complications and Congenital Syndrome}

GBS, acute disseminated encephalomyelitis, myelitis, and other neurological complications, mainly in adults, have been reported following ZIKV infection. The reported incidence of the GBS was higher among males and consistently increased with age. ${ }^{2-4}$

The most striking finding during the ZIKV outbreak in Brazil, however, was the strong cumulative evidence that provided the basis to establish a relationship between ZIKV infection during pregnancy and congenital abnormalities. A wide range of congenital malformations was described, characterized predominantly by CNS alterations, and associated symptoms are microcephaly (with significant cranium-facial disproportion), spasticity, convulsions, marked irritability, and brainstem dysfunction, including feeding difficulties. The results of neuroimaging studies suggest that intrauterine ZIKV infection is associated with severe brain anomalies, such as cerebral calcifications, hydrocephalus, lissencephaly with agenesis of the corpus callosum, pachygyria, cerebellar dysplasia, and white-matter abnormalities ${ }^{2,4}$ (-Fig. 2).

The proportion of affected infants born to mothers infected with ZIKV during pregnancy has not been determined with certainty. Children with congenital syndrome virus face medical and functional challenges that compromise areas of development, some of which become more evident as children age, highlighting the need of early intervention and specialized care from health care providers and caregivers. ${ }^{9}$

The severity of the neurological alterations appears to be related to the period of gestation when the women are infected, that is, the earlier the infection during pregnancy, the more severe the neurologic outcomes to the fetus. Arthrogryposis (-Fig. 3), microphthalmia, funduscopic alterations in the macular region, as well as optic nerve abnormalities were also described in infants with suspected congenital ZIKV syndrome. ${ }^{2,4}$

\section{Treatment and Prevention}

We still do not have any available specific antiviral treatment for patients with ZIKV disease. Only supportive care is indicated, including rest, fluids, and symptomatic treatment (acetaminophen to relieve fever and antihistamines to treat pruritus).

Prevention and control currently rely on personal strategies to avoid mosquito bites and community-level programs to reduce vector densities in endemic areas. Personal

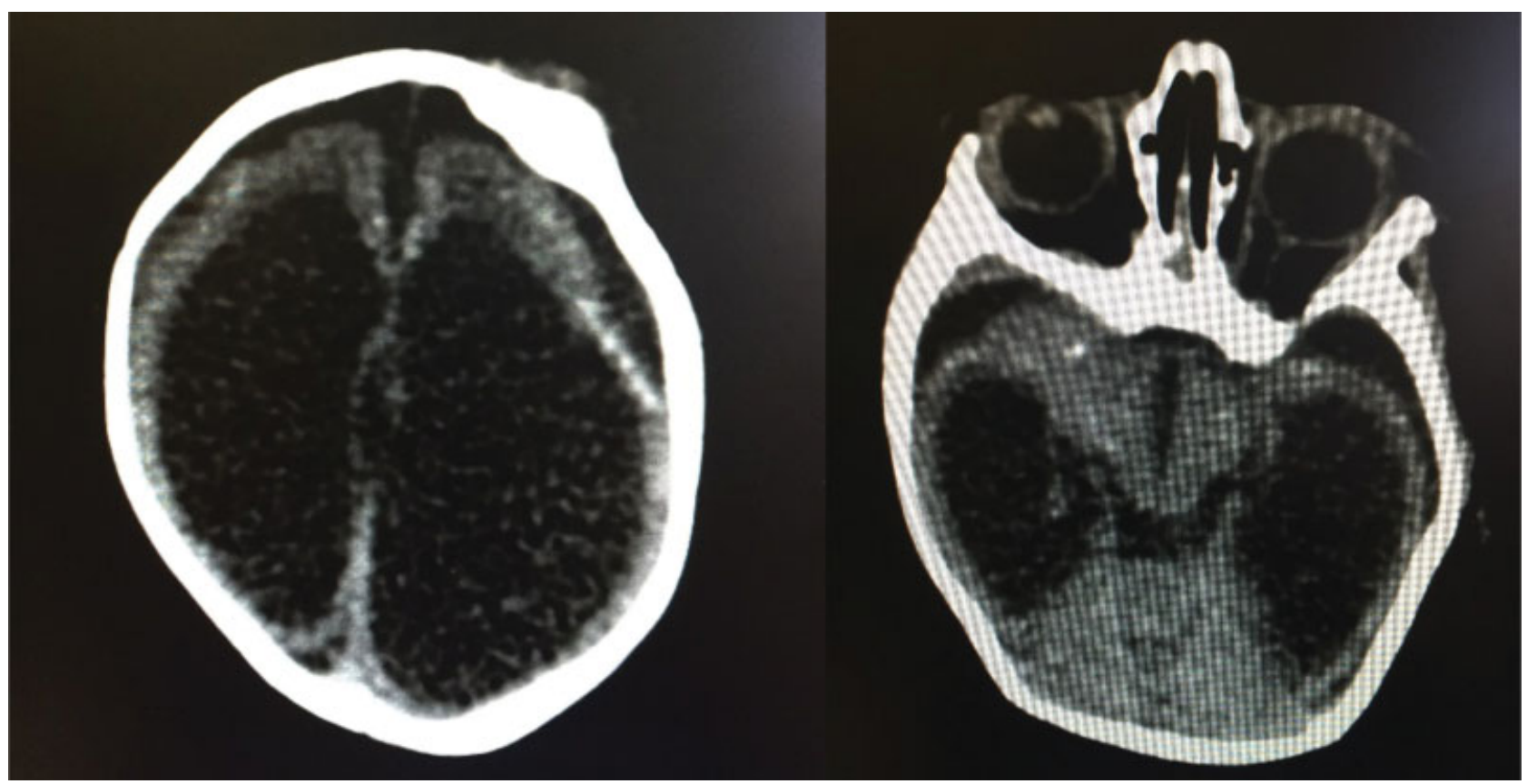

Fig. 2 Head computed tomography scans highlight reduced brain parenchyma and lissencephaly, with dilatation of the infratentorial and supraventricular system. Calcifications, notably in the frontal and parietal lobes. 

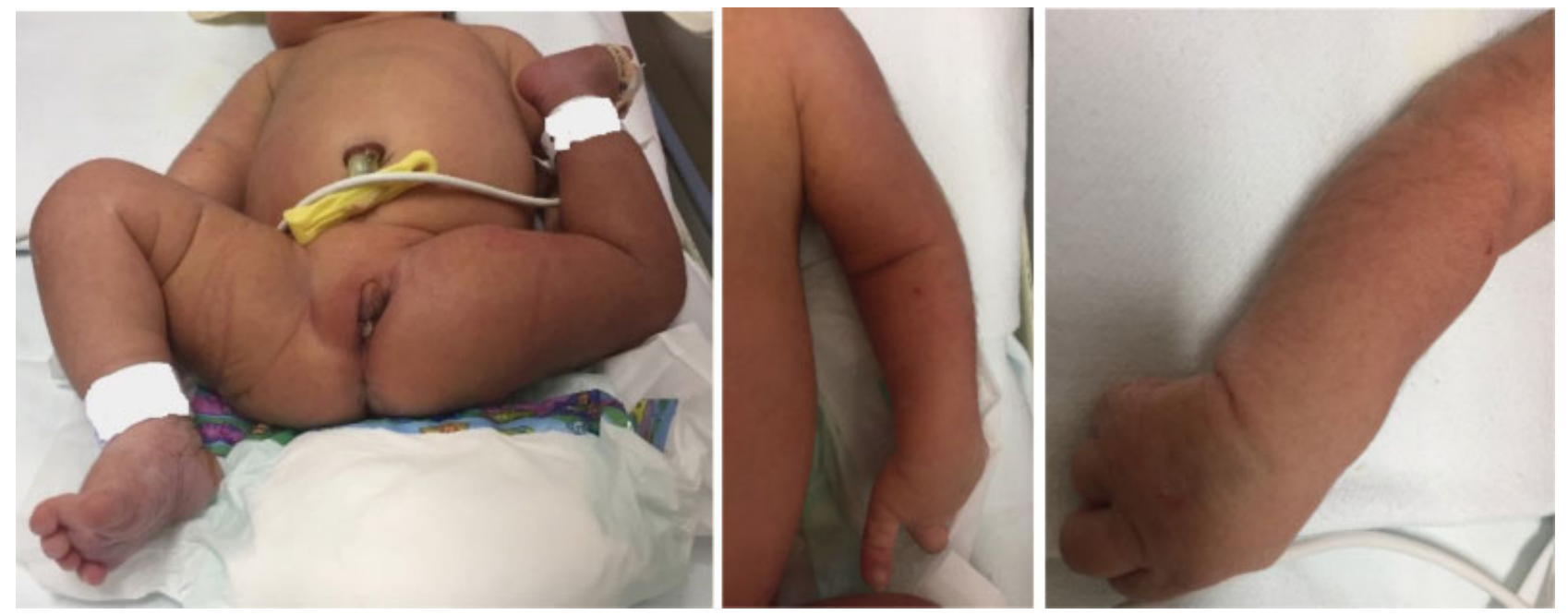

Fig. 3 Arthrogryposis of the hip and wrists.

measures include using insect repellent containing $\mathrm{N}, \mathrm{N}-$ diethyl-meta-toluamide, icaridin, oil of lemon eucalyptus, or ethyl butylacetylaminopropionate. ${ }^{10}$

Preliminary studies identified a single ZIKV serotype and suggested that immune response after ZIKV infection induces broadly neutralizing antibodies against multiple strains (South American, Asian, and early African ZIKV strains proved to be similarly sensitive to neutralization by ZIKV convalescent human serum), paving the way for the development of an effective vaccine. ${ }^{11}$ Similarly to other flaviviruses, neutralizing antibodies appears to play a critical role in protection against infection.

Several ZIKV vaccine candidates using different technologies, based on plasmid DNA, modified mRNA, purified inactivated virus, recombinant live attenuated vaccines, and viral vectored vaccines, showed promising results in mouse and nonhuman primate studies and are now advancing to clinical trials in humans. ${ }^{12}$ Taking in account the need to protect women at childbearing age, vaccination strategies should be prioritized to target individuals of both sexes of reproductive age (to prevent sexual transmission), 9 years of age or older. The recent results with the live-attenuated chimeric dengue vaccine, showing increased risk of severe dengue among dengue-naïve subjects vaccinated compared with the unvaccinated control group, highlights the importance of long-term safety surveillance, to evaluate the duration of the protective immune responses of the current candidate ZIKV vaccines. ${ }^{13}$

The preliminary results of a clinical study with a purified formalin-inactivated ZIKV vaccine and aluminum hydroxide adjuvant were recently published showing that the vaccinated subjects developed neutralizing protective titers that were protective in mice, with only mild and moderate adverse events. Additional inactivated vaccines, proteinbased and peptide-based vaccines are in preclinical development. Several nucleic-acid vaccines are being developed (DNA plasmid vaccine and nucleoside-modified RNA vaccine against the ZIKV, which expresses the premembrane and envelope proteins), as well as vectored vaccines that use adenovirus, vesicular stomatitis virus, measles, and other backbones, including a live attenuated vaccine based on the tetravalent dengue vaccine developed by the U.S. National Institutes of Health and the Butantan Institute in Brazil. ${ }^{14}$

Although DNA vaccines, as well as subunit vaccines are safe and potentially easily manufactured, they have limited immunogenicity comparing to other vaccine types, such as live-attenuated vaccines. ${ }^{14}$

Even though promising progress has been made in the area of research and development of candidate vaccines, several challenges lie ahead. It is crucial for a ZIKV vaccine efficacy phase III trial to have clear clinical case definitions and accurate laboratory tests to allow case ascertainment on suspected cases. It will be also critical, when planning vaccine trials, a better knowledge on the correlates of protection and on the immune responses after subsequent infections with these flaviviruses. It is still unknown whether a previous infection with other flaviviruses, like dengue, or the presence of antibodies against yellow fever in populations where vaccination is routinely recommended, will impact on the risk of severe disease, neurological complications like GBS, or congenital disease in pregnant women. Vaccination should not trigger the development of GBS and other autoimmune complications are already demonstrated to occur in higher risk after ZIKV infection. More importantly, the current epidemiological global situations in endemic areas, with a substantial decrease in the number of cases reported jeopardize the feasibility of phase-3 studies to evaluate the efficacy of these candidate vaccines.

\section{Funding}

None.

Conflict of Interest

None declared.

\section{References}

1 Musso D, Gubler DJ. Zika Virus. Clin Microbiol Rev 2016;29(03): 487-524

2 Sáfadi MAP, Nascimento-Carvalho CM. Update on Zika: what you need to know. Pediatr Infect Dis J 2017;36(03):333-336 
3 Cauchemez S, Besnard M, Bompard P, et al. Association between Zika virus and microcephaly in French Polynesia, 2013-15: a retrospective study. Lancet 2016;387(10033):2125-2132

4 CDC. Zika virus. Available at: https://www.cdc.gov/zika/index. html. Accessed March 27, 2019

5 .Ministério da Saúde. Boletins epidemiológicos. Available at: http:// portalms.saude.gov.br/boletins-epidemiologicos. Accessed March 23, 2019

6 Secretaria de Vigilância em Saúde; Ministério da Saúde; Situação epidemiológica. Monitoramento integrado de alterações no crescimento e desenvolvimento relacionadas à infecção pelo vírus Zika e outras etiologias infecciosas, até a Semana Epidemiológica 52 de 2018. Boletim Epidemiológico 2019;50(08):1-8 Available at: http://portalarquivos2.saude.gov.br/images/pdf/2019/marco/ 22/2019-001.pdf

7 Snyder RE, Boone CE, Cardoso CAA, Aguiar-Alves F, Neves FPG, Riley LW.Zika: A scourge in urban slums. PLoS Negl Trop Dis 2017; 11(03):e0005287

8 Oliveira DB, Almeida FJ, Durigon EL, et al. Prolonged shedding of Zika virus associated with congenital infection. N Engl J Med 2016;375(12):1202-1204
9 Satterfield-Nash A, Kotzky K, Allen J, et al. Health and development at age 19-24 months of 19 children who were born with microcephaly and laboratory evidence of congenital Zika virus infection during the 2015 Zika virus outbreak Brazil, 2017. MMWR Morb Mortal Wkly Rep 2017;66(49): 1347-1351

10 American Academy of Pediatrics. Zika Virus. In: Kimberlin DW, Brady MT, Jackson MA, Long SS, eds. Red Book: 2018-2021 Report of the Committee on Infectious Disease. 31st ed. Elk Grove Village, IL: American Academy of Pediatrics; 2018:894-901

11 Dowd KA, DeMaso CR, Pelc RS, et al. Broadly neutralizing activity of zika virus-immune sera identifies a single viral serotype. Cell Reports 2016;16(06):1485-1491

12 Poland GA, Kennedy RB, Ovsyannikova IG, Palacios R, Ho PL, Kalil J. Development of vaccines against Zika virus. Lancet Infect Dis 2018;18(07):e211-e219

13 Halstead SB. Achieving safe, effective, and durable Zika virus vaccines: lessons from dengue. Lancet Infect Dis 2017;17(11): e378-e382

14 Durbin A, Wilder-Smith A. An update on Zika vaccine developments. Expert Rev Vaccines 2017;16(08):781-787 